\title{
Family based colorectal cancer screening in a district hospital
}

\author{
N P J Cripps, R J Heald
}

\begin{abstract}
Colorectal cancer (CRC) screening using family history to define a group at increased risk is gaining support. Three hundred and ninety six subjects aged over 25 with at least one first degree affected relative have been screened using a single slide, immunological faecal occult blood test (FOBT), and family history data to select the highest risk group. Compliance was $64.9 \%$ but was significantly better if contact was made within one year of diagnosis of the index relative $(75 \% v 62.1 \%$, $\left.\chi^{2}=5 \cdot 7, p<0 \cdot 05\right)$. Twelve subjects $(13 \cdot 2 \%)$ of those who had a colonoscopy) bearing adenomas have been detected, three of which were at high risk of malignant transformation. No cancers have been diagnosed. Most subjects undergoing screening were less than 55 years of age $(67 \cdot 8 \%)$. These accounted for most colonoscopies $(68 \cdot 1 \%)$ but only one large adenoma was diagnosed in this group. The FOBT was particularly useful, enabling the detection of five large adenomas. Family based CRC screening is practical in a district hospital setting. Although labour intensive, it meets a population demand and can detect significant numbers of adenomas. Screening, even in those at moderately increased risk, could be focused on the older age groups, probably those aged over 40 .

(Gut 1996; 38: 421-425)
\end{abstract}

Keywords: colorectal cancer, family history, screening.

Familial clustering of colorectal cancer (CRC) was first reported in $1904 .{ }^{1}$ Lovett identified a two to fourfold increase in the expected death rate in a study of mortality cause among first degree relatives of CRC patients. ${ }^{2}$ The CRC incidence among relatives of patients affected by apparently sporadic disease has similarly been shown to be four times that expected. ${ }^{3}$ Screening studies in Israel, ${ }^{4}$ in the USA, ${ }^{56}$ and in the United Kingdom, ${ }^{78}$ have confirmed the tendency for a familial inheritance of colorectal adenomas in those not affected by well defined CRC syndromes.

Most screening protocols for subjects at increased risk because of a family history of CRC include faecal occult blood testing (FOBT) and flexible sigmoidoscopy for all. If a particularly strong family history is evident, ${ }^{48}$ colonoscopy is the first choice investigation because of the increased prevalence of right colonic neoplasia in dominantly inherited pedigrees. $^{7}$ Colonoscopy is the first choice investigation after positive FOBTs.
We have been offering screening to subjects with first degree relatives affected by CRC since 1992. We aimed to establish the significance of a family history of CRC in asymptomatic relatives in a district hospital and, in this setting, to establish if screening asymptomatic family members from 25 years is worthwhile. We have attempted to validate family history based screening in a protocol that does not include flexible sigmoidoscopy for all those screened. We have tried to inform those with a family history of CRC of its significance and we have informed them of the important symptoms of the disease.

\section{Methods}

Potential subjects for screening over age 25 were identified by contacting relatives of past CRC patients whose details have been maintained on a database since 1979. At first, contacts were made for those still living but later, relatives of dead patients were included. Index subjects were initially chosen at random. Later, those who had developed the disease aged less than 45 were targeted; the relatives of young index cases are at relatively high risk and we aimed to contact people expected to gain most from screening (Table I). ${ }^{7}$

One postal approach was made to each person. Subjects willing to be screened contacted the office to request a symptom based questionnaire and a FOBT kit. A leaflet describing those at increased risk of CRC and the method of screening was sent with the letter of introduction. ${ }^{9}$

Questionnaire responses provided family history details for CRC and other malignancy; answers to other questions reported bowel symptoms. A single slide HemeSelect FOBT (SmithKline Diagnostics, San Jose, CA) was completed and returned. The lifetime risk of developing CRC was estimated from family history data (Table I). ${ }^{7}$

Table II summarises the screening policy. If lifetime risk was $<1: 10$, the FOBT negative

TABLE I The extent of family history and estimated lifetime risk

\begin{tabular}{ll}
\hline Number of affected relatives & Attributed lifetime risk \\
\hline No affected relatives & $1: 50$ \\
1 first degree relative & $1: 17$ \\
1 first and 1 second degree relative $\dagger$ & $1: 12$ \\
1 first degree relative age $<45$ years & $1: 10$ \\
Both parents affected & $1: 8 \cdot 5$ \\
2 first degree relatives $\ddagger$ & $1: 6$ \\
3 first degree relatives & $1: 2$ \\
\hline
\end{tabular}

The estimated lifetime risk is shown according to the number of affected relatives in the pedigree. Three affected first degree relatives are sufficient to suspect a dominant inheritance pattern. The background lifetime risk is $1: 50 .^{7 \star}$ Parent, sibling, etc; tuncle, grandparent, etc; $\neq$ not both parents.

Colorectal Research
Unit, North
Hampshire Hospitals
NHS Trust,
Basingstoke,
Hampshire
N P J Cripps
R J Heald
Correspondence to:
Mr N P Cripps, Colorectal
Research Unit, 'C' Floor,
North Hampshire Hospitals
NHS Trust, Aldermaston
Road, Basingstoke,
Hampshire RG24 9NA.
Accepted for publication
7 September 1995


TABLE II Family history and FOBT based screening protocol

Lifetime risk $\geqslant 1: 10$

FOBT positive

Significant symptoms alone

\section{Colonoscopy Colonoscopy Interview and rigid sigmoidoscopy}

The decision to recommend colonoscopy is based on the estimated lifetime risk and the result of the FOBT. Assuming no other factors were significant, relevant symptoms led to an outpatient style interview.

and no symptoms reported, subjects were advised that their immediate risk of developing CRC was not excessive and that further testing was unnecessary. All responders were told that a further screening opportunity would be offered two years later. Each was reminded that all bowel symptoms - particularly rectal bleeding - should be reported to a doctor. This was emphasised because screening had not physically excluded neoplasia in people at a moderately increased risk of developing CRC.

This report deals with the results of a CRC screening study at a single point in the lifetime of subjects considered to be at a higher than average risk of developing the disease. Because the inherited increased risk is effective over the whole lifetime, the true impact of such screening will only be seen after a much longer time interval. The results of the study are presented with this in mind.

\section{Results}

\section{Subjects screened}

Figure 1 shows the age proportions of the subjects who were screened. All patients have had only one colonoscopy to date; the number of colonoscopies undertaken in each age group is shown in the Figure. Figure 2 shows the source of subjects. A total of 482 relatives were identified from 179 index cases and 127 others enrolled by different routes. Local publicity resulted in $59(9.7 \%)$ people referred by their general practitioners, $36(5.9 \%)$ people requested screening after reading the leaflet, and $32(5 \cdot 3 \%)$ were enrolled at the request of hospital clinicians.

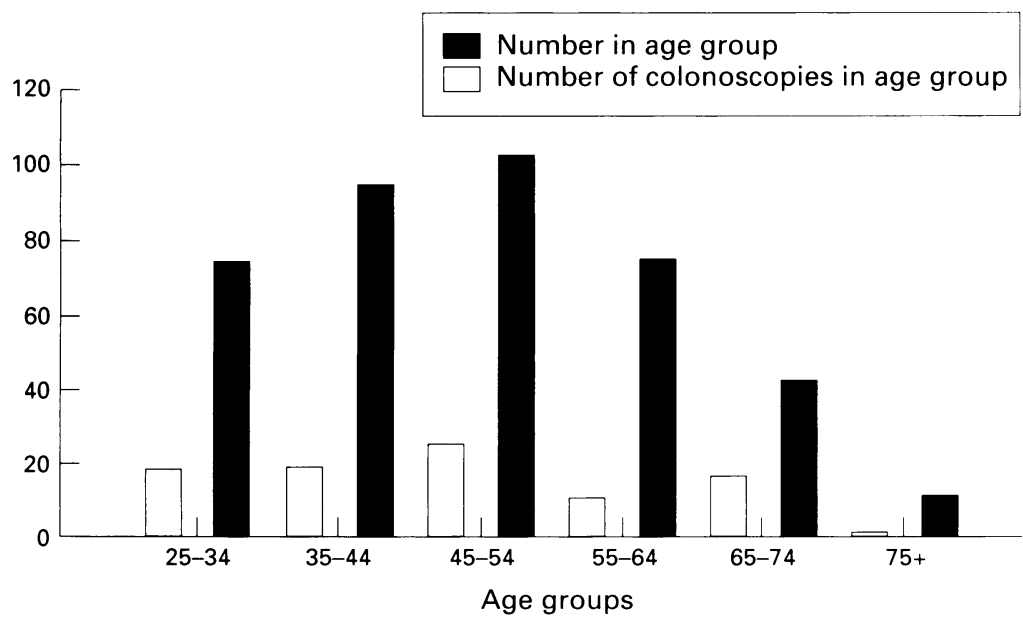

Figure 1: Age distribution of the screened population. Some $67 \cdot 8 \%$ of those screened were younger than 55 years old. This age group accounted for $68.1 \%$ of colonoscopies.

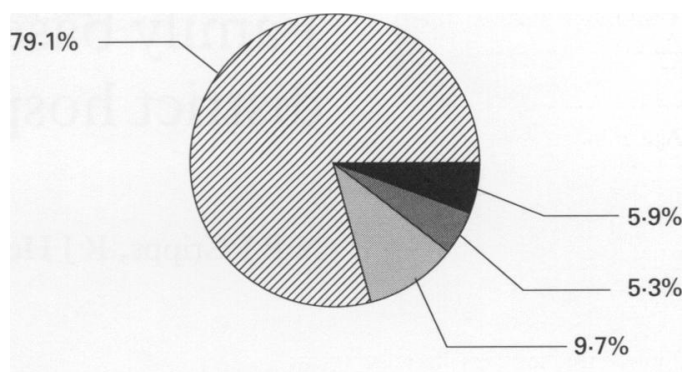

Source of screened population

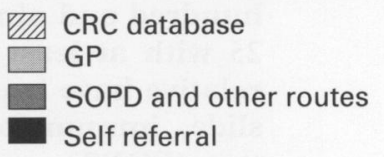

Figure 2: Source of study population. The largest proportion of people have been contacted via their relatives on the colorectal cancer database. The proportions of those from other sources gradually increased as screening progressed. SOPD = surgical outpatient department.

\section{Lifetime risk}

Figure 3 shows the breakdown of lifetime risk. Some $59 \cdot 6 \%$ of those who completed the protocol had only one affected relative. The smallest group contained people with three or more affected first degree relatives; only four subjects from two families were potentially in a dominant pedigree.

\section{Compliance with screening}

Compliance improved as more volunteers were enrolled but was consistently in the region of 60\%. Between April 1992 and September 1994, 396 (64.9\%) people completed the protocol. One hundred and twenty five people $(20.4 \%)$ did not respond to the

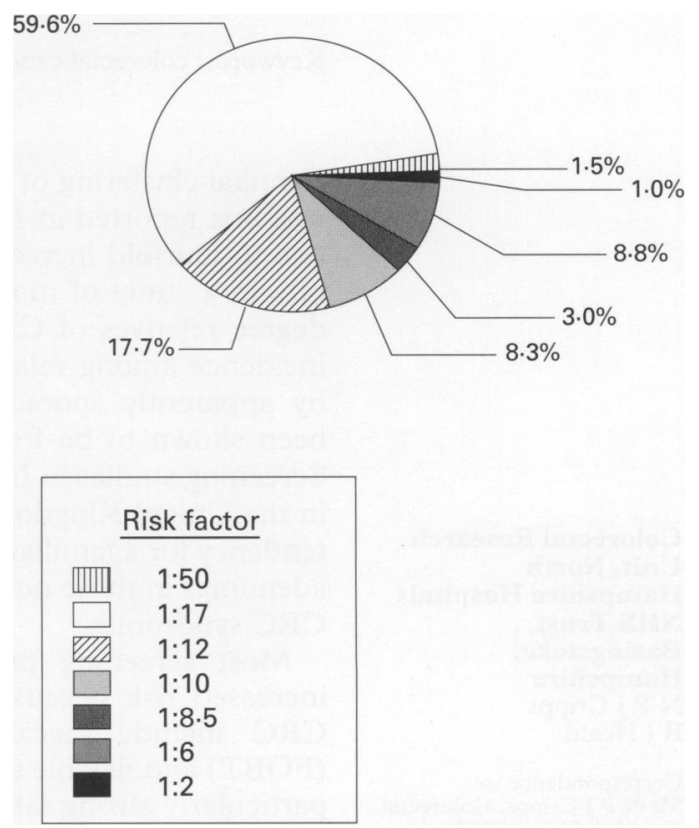

Figure 3: Estimated lifetime risk. A small number of people were found not to have any family history of CRC. Fust under a quarter of those screened had a lifetime risk of $\geqslant 1: 10$, which justified colonoscopy. By this criterion, however, only four people might have been in a dominant pedigree. 
TABLE III Factors affecting compliance with screening

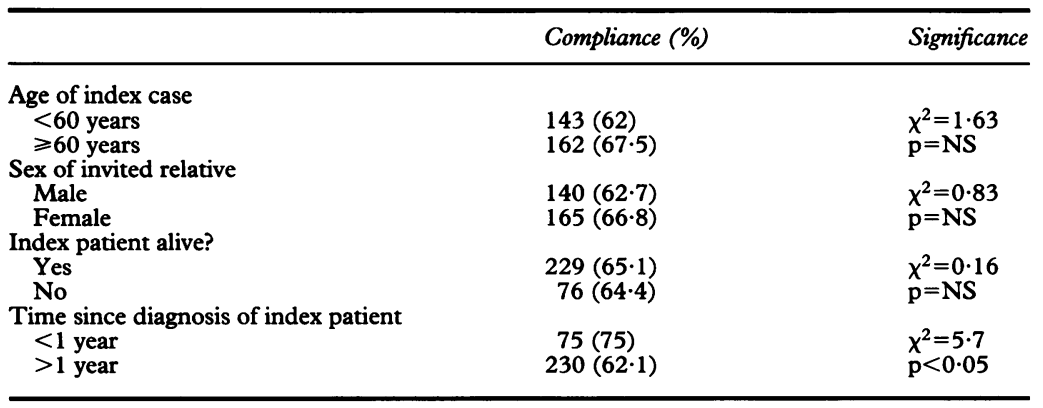

The only factor that significantly affected compliance was the time since diagnosis of the index patient. screening invitation. Eighty nine (14.6\%) defaulted between requesting and returning the questionnaire and FOBT - this added to screening costs because these FOBT kits were lost.

We could not define the characteristics of the non-compliant group because detailed demographic information could only be obtained when subjects had completed the protocol. Table III shows details of the index cases in relation to compliance. Time since diagnosis of the index case was the only factor to significantly affect uptake, probably because of influence by the affected person.

\section{Outcome of colonoscopy}

Total colonoscopy was achieved in $85(93.4 \%)$ cases. Double contrast barium enema was used after incomplete examination. A 74 year old with 10 left sided colorectal polyps (mixed adenomatous and metaplastic) has refused further examination. One 69 year old had sigmoid diverticular disease that precluded total colonoscopy; otherwise examination was to the splenic flexure, or further, in all cases. Only histological proof confirmed adenoma presence. One postpolypectomy haemorrhage required a two unit blood transfusion, otherwise no morbidity was caused by screening.

\section{FOBT results}

Twenty four $(6 \cdot 1 \%)$ HemeSelect slides were positive. One hundred colonoscopies were indicated by the protocol and 91 have been completed. In addition to those for positive
FOBTs, 78 were indicated by lifetime risk criteria and two for both reasons.

Adenomas were diagnosed in six of 20 colonoscopies for positive FOBT contrasting with adenomas found in six of 71 colonoscopies for lifetime risk $\left(\chi^{2}=4.59\right.$ with Yates's correction; 1 df, $p<0.05)$. Five of six adenomas discovered by occult blood testing were larger than $1 \mathrm{~cm}$ compared with two of six adenomas in the group examined for excessive lifetime risk.

\section{Screen detected neoplasia}

Twelve people (13.2\% of colonoscopies) have been diagnosed with colorectal adenomas. No cancers have been detected. Another $2 \mathrm{~cm}$ adenoma has been diagnosed elsewhere in a relative of a database patient. Table IV shows the characteristics of the adenomas detected by our programme.

If single adenomas less than $1 \mathrm{~cm}$ in size have minimal risk of malignancy or early malignant transformation, seven of 12 adenomas detected were of immediate clinical significance. Most important is the 64 year old diagnosed with familial adenomatous polyposis who had one first degree relative affected by both CRC and periampullary carcinoma. This patient had a large caecal villous adenoma and more than 300 smaller adenomas and, after surgery, has been counselled by a medical geneticist. Two people, aged 65 and 68, had dysplastic rectal adenomas larger than $5 \mathrm{~cm}$.

One of four adenomas diagnosed in those younger than 55 was larger than $1 \mathrm{~cm}$. Sixty three colonoscopies carried out in this age group represented $68 \cdot 1 \%$ of the endoscopic workload. Six of eight adenomas detected in those older than 55 were larger than $1 \mathrm{~cm}$ and only 28 colonoscopies were needed to diagnose these.

One adenoma (which was diminutive) would not have been detected by flexible sigmoidoscopy. This 31 year old had recorded a negative FOBT but was examined because of a mistaken interpretation of her estimated lifetime risk on initial assessment, which was subsequently revised. All other subjects had adenomas that would readily have been diagnosed by flexible sigmoidoscopy.

\section{Discussion}

We have deviated from the usual family history based screening protocol, largely because of flaws in the chemistry of guaiac FOBTs that limit their sensitivity and specificity. 1011 Haemoccult has performed poorly in population based screening studies detecting 38-50\% of cancers in recent large trials. ${ }^{12-14}$ Although $74 \%$ of colorectal cancers were detected in the population based Nottingham trial, ${ }^{15}$ its ability to detect colorectal adenomas is poor. ${ }^{14}$ Adenoma prediction has also been disappointing in family history based screening studies; Houlston $^{7}$ and Stephenson ${ }^{8}$ found poor negative predictive values for adenoma of $78 \%$ and $87 \%$ respectively. HemeSelect and Haemoccult were recently compared in a large group 
TABLE $\mathrm{V}$ The population and study incidence of colorectal adenoma

\begin{tabular}{rllll}
\hline Age & $\begin{array}{l}\text { Percentage } \\
\text { expected with }\end{array}$ & $\begin{array}{l}\text { Number in } \\
\text { current study }\end{array}$ & $\begin{array}{l}\text { Number of } \\
\text { patients with } \\
\text { adenoma } \\
\text { expected }\end{array}$ & $\begin{array}{l}\text { Number of } \\
\text { patients with } \\
\text { adenoma in } \\
\text { study }\end{array}$ \\
\hline$<40$ & 4 & 120 & 5 & 2 \\
$40-49$ & 3 & 118 & 4 & 2 \\
$50-59$ & 9 & 78 & 7 & 0 \\
$60-69$ & 11 & 52 & 6 & 7 \\
$\geqslant 70$ & 10 & 27 & 3 & 1 \\
\hline
\end{tabular}

Data taken from Atkin, ${ }^{21}$ compiled from refs $\mathrm{McCallum}$, Rosevelt and Shida, which described the flexible sigmoidoscopic yield in 3392 average risk patients.

of randomly selected $50-75$ year olds. Positive predictive values for carcinoma and adenoma were similar for both tests but Haemoccult detected only one of nine carcinomas detected by HemeSelect. Seven large adenomas were detected by Haemoccult compared with 31 detected by HemeSelect. ${ }^{16}$ Additional endoscopic work resulting from the more sensitive immunological test may be justified by improved survival after screening.

Compliance with FOBT screening is poor, with $38-53 \%$ of average risk subjects ${ }^{14} 15$ and $52 \cdot 2 \%$ of high risk siblings ${ }^{17}$ some examples of uptake. Compliance was reduced from $88 \%$ to $63 \%$ when a single and a two day slide test were compared, with a corresponding fall in the positive test rate from $3 \cdot 7 \%$ to $2 \cdot 6 \%$. (T Aisawa et al, Proceedings of 8th AsianPacific Congress of Gastroenterology, 1988.) One and three day HemeSelect regimens were recently compared in the United Kingdom without significant difference in compliance, although participation of $43-46 \%$ was poor - a finding put down to the complexity of the instructions for stool testing. ${ }^{16} \mathrm{We}$ aimed to use a single slide FOBT to maximise compliance, a policy that seems justified by our acceptance rate.

Without HemeSelect, only four small and one large adenoma would have been detected by our protocol. The FOBT detected five significantly sized adenomas with a positive predictive value of $30 \%$, a positive test rate of $6 \cdot 1 \%$, acceptable compliance, and a minimal approach to those screened. Our results for the performance of HemeSelect are in keeping with the findings of other investigators. ${ }^{16}$ Although HemeSelect is unproved in screening for asymptomatic colonic neoplasia, to commence screening by our protocol using a guaiac FOBT would have risked missing significant abnormality in those with a single first degree affected relative.

We have also deviated from the format of other screening programmes in the choice of colonoscopy rather than flexible sigmoidoscopy as the mainstay of colonic examination. Others have shown that flexible sigmoidoscopy is satisfactory for colonic examination in those with only one first degree relative. ${ }^{18} 19 \mathrm{We}$ expected the pattern of family history to resemble that of the St Mark's Hospital family cancer screening clinic where people with young affected relatives and those with more than one affected family member were specifically targeted. The Guildford survey aimed at screening a similar population and found 65 subjects from 43 families in possible dominant pedigrees. ${ }^{20}$ We also sought to include those with higher lifetime risks and were surprised to find that so few of our group were in such pedigrees. The difference between the two studies is dramatic and may warrant further analysis, but despite this, the adenoma yield was similar. We now agree that it would have been reasonable to use flexible sigmoidoscopy as the usual method of endoscopy in all subjects (except for a positive FOBT or dominant pedigree), with follow up colonoscopy as appropriate. This approach would have been easier and cheaper in terms of endoscopic commitment.

The age at which to begin screening is somewhat arbitrary. We chose age 25 and Carpenter chose age $30 .{ }^{20} \mathrm{We}$ started at this age because of our expectations of the likely family history pattern, which did not materialise. Outwith dominant pedigrees, we now consider age 40 (or 15 years younger than the index case) to be appropriate to start endoscopic screening; below this age CRC is rare.

Adenomas were diagnosed in 12 of 91 subjects who had a colonoscopy $(13 \cdot 2 \%)$. The age range of the study group indicates that approximately 25 subjects would have been expected to bear adenomas (Table V). ${ }^{21}$ It is difficult to predict the number of large adenomas expected in our population. The youth of the screened group may explain why so few were detected. We have, however, diagnosed at least three people who had adenomas likely to undergo early malignant transformation. We were not surprised that a carcinoma was not found in this small group.

These results provide evidence to support the further assessment of the immunological FOBT in CRC screening, perhaps as a comparison between single and three slide tests. We are considering redirecting money saved by undertaking fewer colonoscopies to more frequent - that is, - annual FOB testing.

There is a demand, among first degree relatives of CRC patients, for counselling and examination to assess their risk of developing the disease. ${ }^{20}$ The well defined CRC syndromes make up only $10-20 \%$ of all cases and advances in genetic testing are unlikely to have an immediate impact on the incidence of most cancers. Until the familial colon cancer genes are further characterised, genetic screening for families and others not in definite inheritance pedigrees is an unrealised objective. Demand for programmes to identify and treat these patients will remain. ${ }^{22}$ Our study, and the Guildford one, ${ }^{20}$ will have included examples of sporadic CRC despite attempts to target

TABLE VI Recommendations for family history based CRC screening

Begin screening at age 40 (or 15 years younger than index case) unless in a dominant pedigree

Contact relatives soon after diagnosis of the index case to maximise compliance

Choose flexible sigmoidoscopy as mainstay of colonic examination except after positive FOBTs and in potentially dominant pedigrees

Use a specific (immunological) FOBT

Recommendations for screening for CRC in a group at increased risk due to a family history of the disease. 
high risk pedigrees. For this reason, family screening clinics will have a limited impact on sporadic CRC and the role of the specific FOBT in, and outside, such protocols is especially important.

The cost of screening to date, including secretarial support, HemeSelect tests, and colonoscopy is estimated at $£ 19630$. Each adenoma detected cost approximately $£ 1630$ (or $£ 6540$ for each of the three more significant adenomas). The major expenditure was for day case colonoscopy, which could have been reduced had flexible sigmoidoscopy been preferred for colonic examination. These costs are similar to those incurred at Guildford and the low yield from this outlay is comparable. ${ }^{20}$ Similarly, the benefit of reassurance given to a large number of people is unquantifiable.

We have screened first degree relatives of subjects affected by CRC in a district hospital setting, using a novel protocol to good effect. In the light of our results, some recommendations for others proposing to establish a similar service are shown in Table VI. Family based CRC screening in a district hospital setting meets a population demand and has the capacity to detect significant abnormalities.

This project owes its existence to generous financial support from the Wessex Cancer Trust; without this help it would have been impossible to begin screening.

been impossible to begin screening. The invaluable assistance of Mrs J Wilson and Mrs R Sexton
in the day to day administration of the screening project is gratefully acknowledged.

1 Watkins DJG. A family tree. BMF 1904; i: 190.

2 Lovett E. Family studies in cancer of the colon and rectum. Brf Surg 1976; 63: 13-8.

3 Stephenson BM, Finan PJ, Gascoyne J, Garbett F, Murday VA, Bishop DT. Frequency of familial colorectal cancer. Br $\mathcal{F}$ Surg 1991; 78: $1162-6$.

4 Rozen P, Fireman ZVI, Figer A, Legum C, Ron E, Lynch H. Family history of colorectal cancer as a marker of
. Fozen P, Fireman ZVI, Figer A, Legum C, Ron E, Lynch potential malignancy within a screening programme. Cancer 1987; 60: 248-54.
5 Cannon-Albright LA, Skolnick MH, Bishop T, Lee RG, Burt RW. Common inheritance of susceptibility to colonic adenomatous polyps and associated colorectal cancers. N Engl f Med 1988; 319: 533-7.

6 Guillem JG, Neugut AI, Forde KA, Waye JD, Treat MR. Colonic neoplasms in asymptomatic first-degree relatives of colon cancer patients. Am f Gastroenterol 1988; 83: of colon

7 Houlston RS, Murday V, Haracopos C, Williams CB, Slack J. Screening and genetic counselling for relatives of patients with colorectal cancer in a family cancer clinic. BMF 1990; 301: 366-8.

8 Stephenson BM, Murday VA, Finan PJ, Quirke P, Dixon MF, Bishop DT. Feasibility of family based screening for colorectal neoplasia: experience in one general surgical practice. Gut 1993; 34: 96-100.

9 Heald RJ, Cripps NPJ, Basingstoke family bowel cancer screening programme. No 18 in a series of help yourself leaflets. 11 Westwood Road,

10 St John DJB. Faecal occult blood tests: a critical review. In: Hardcastle JD, ed. Screening for colorectal cancer. Engelwood NJ: Normed Verlog, 1989: 54-68.

11 Eddy DM. Screening for colorectal cancer. Ann Internal Med 1990; 113: 373-84.

12 Kronborg O, Fenger C, Olsen J, Bech K, Sondergaard O. Repeated screening for colorectal cancer with fecal occult blood test: a prospective randomised study at Funen, Denmark. Scand F Gastroenterol 1989; 24: 599-606.

13 Kewenter J, Bjork S, Haglind E, Smith L, Svanvik J, Ahren C. Screening and rescreening for colorectal cancer in 27700 subjects. Cancer 1988; 62: 645-51.

14 Foley DP, Dunne P, Dervan PJ, Callaghan TWO, Crowe J, Lennon JR. Left sided colonoscopy and haemoccult Lennon JR. Left sided colonoscopy and haemoccult screening for colorectal neoplasia. European four
Gastroenterology and Hepatology 1992; 4: 925-35.

15 Hardcastle JD, Thomas WM, Chamberlain J, Pye G, Sheffield J, James PD, et al. Randomised controlled trial of faecal occult blood screening for colorectal cancer. The results of the first 107349 subjects. Lancet 1989; i: $1160-4$.

16 Robinson MHE, Marks CG, Farrands PA, Thomas WM, Hardcastle JD. Population screening for colorectal cancer: comparison between guaiac and immunological faecal comparison between guaiac and immuno-ogical

17 Sandler RS, DeVellis BM, Blalock SJ, Holland KL. Participation of high-risk subjects in colon cancer screen-

18 Grossman S, Milos M. Colonoscopic screening of persons with suspected risk factors for colon cancer. I. Family history. Gastroenterology 1988; 94: 395-400.

19 McConnell JC, Nizin JS, Slade MS. Colonoscopy in patients with a primary family history of colon cancer. Dis Colon Rectum 1990; 33: 105-7.

20 Carpenter S, Broughton M, Marks CG. A screening clinic for relatives of patients with colorectal cancer in a district general hospital. Gut 1995; 36: 90-2.

21 Atkin WS, Cuzick J, Northover JMA, Whynes DK. Prevention of colorectal cancer by once-only sigmoidoscopy. Lancet 1993; 341: 736-40.

22 Thomas HJW. Familial colorectal cancer. BMf 1993; 307: 277-8. 